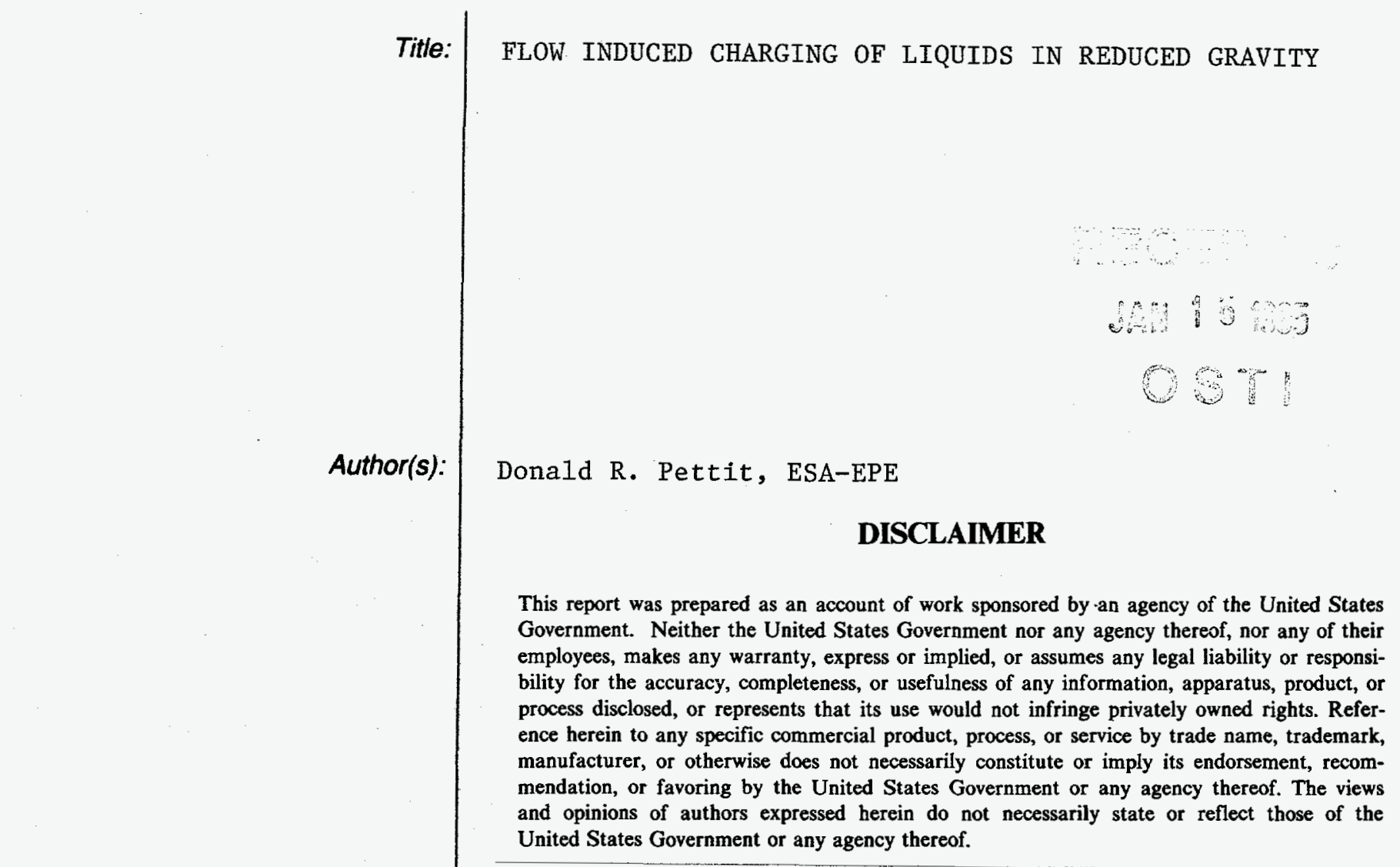

Submitted to: Space ' 96 Conference

Albuquerque, NM

June $1-5,1996$

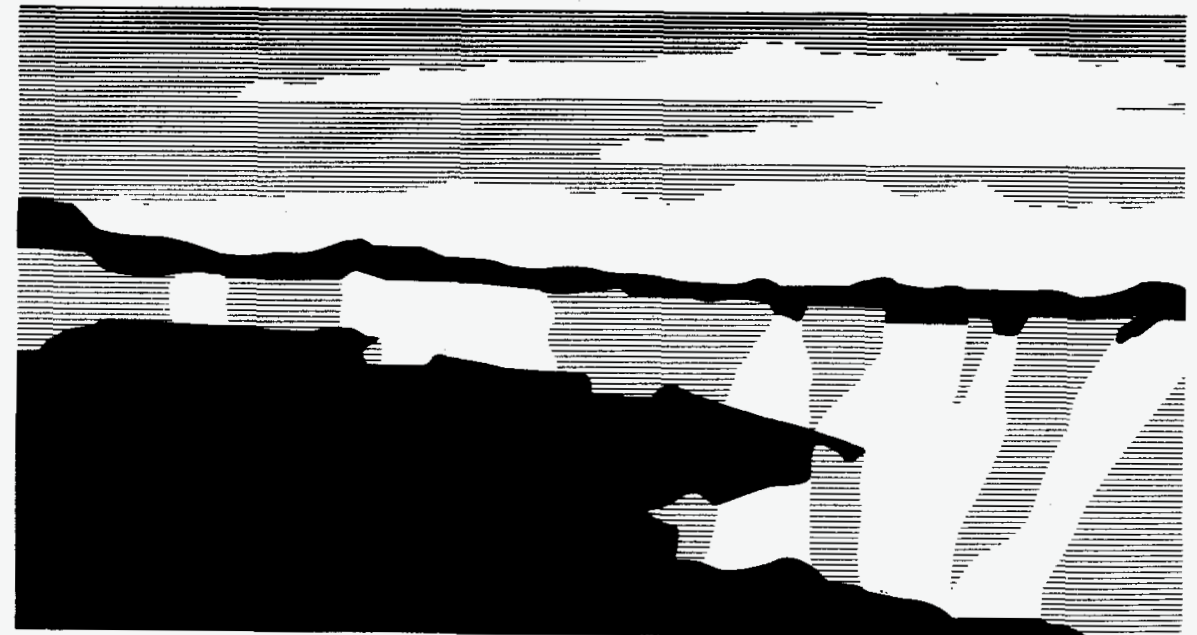




\title{
Flow Induced Charging of Liquids in Reduced Gravity
}

\author{
Donald Pettit \\ Los Alamos National Laboratory \\ Los Alamos, New Mexico \\ dpettit@lanl.gov
}

\begin{abstract}
Microgravity experiments on free fluid surfaces of large length scale could be subject to experimental artifact from flow induced charging. Under conditions favorable for flow induced charging, flowing liquids develop a static electrical charge which manifests itself as a force whose magnitude approaches that of surface tension force. Favorable conditions are: a non-conducting liquid, a small diameter non-conducting flow passage, a large flow volume, and a small separation distance between the fluid and another object. We present a method for calculating the magnitude of flow induced charging and scaling arguments so that potential problems can be determined and dealt with at the experimental design phase. A dimensionless ratio of charge force to surface tension force we call the Hula Number should be less than 0.5 to prevent artifact or unwanted fluid motion.
\end{abstract}

\section{Introduction}

We are all familiar with scuffing our feet on the rug and touching the unsuspecting dog on the nose (obviously, a lad with a bright future in experimental science, albeit missing a finger). There is a fluid dynamic analog to this mechanical static electric generation called flow induced charging. Fluid friction between an electrically non-conducting wall and an electrically nonconducting flowing fluid can build up sizable static charges that flow with the liquid. For example, flowing petroleum based fuels in rubber hoses have been known to create sufficient charge to release a volley of sparks which can cause fire and explosion, hence, fueling operations generally have a grounding strap. Under certain conditions, electrochemical corrosion of pipelines can be accelerated by flow induced charging. Apart from a few known problems which are handled with rule-of-thumb engineering, flow induced charging remains rather obscure ${ }^{1}$.

Fluid dynamic experiments performed in the reduced gravity environment of orbit often center around observing second or third order forces that are masked by gravitational force if done on Earth. Surface tension experiments would be one example. If the fluid for the experiment is injected into the apparatus under conditions favorable for flow induced charging, the resulting 
charge forces could be of the same order of magnitude as the other forces that are under investigation. This could introduce considerable artifact or possibly cause complete failure due to the fluid not behaving as it was suppose to ${ }^{2}$. In this paper we develop a method for calculating the magnitude of flow induced charging and scaling arguments so that potential problems can be determined and dealt with at the experimental design phase.

\section{Theory}

A solid-liquid interface usually has an adsorbed double layer of electric charge $^{3}$. Consider the outer or diffuse part of the Stern electric double layer within a fluid at a distance $\tau$ from a stationary, electrically non-conducting wall with some geometric length scale $r$ as shown in Figure 1. For $r$ of the order of millimeters and $\tau$ of the order of 10's $\mathrm{nm}$, we assume this charge layer to be described by flat geometry $(\tau<<<r)$.

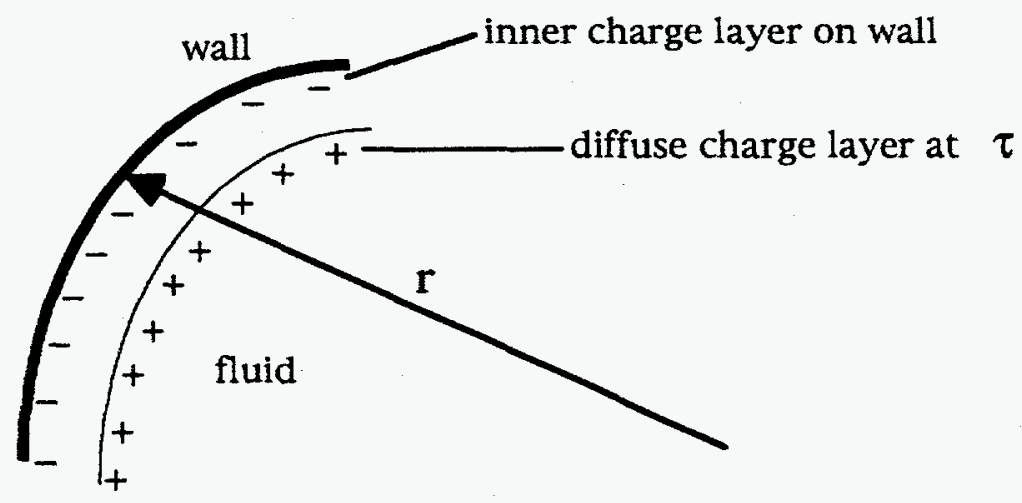

Figure 1

Let $q$ be the charge (coul), $V$ be the voltage (volts or $N t-m / c o u l)$ and $C_{p}$ be the capacitance (coul/volt or coul $2 / \mathrm{Nt}-\mathrm{m}$ ); then $\mathrm{q}=\mathrm{VC} \mathrm{p}$. For flat plates, $\mathrm{C}_{\mathrm{p}}=\mathrm{D} \varepsilon_{0} \mathrm{~A} / \mathrm{d}$, where $D$ is the dielectric constant (unitless), $\varepsilon_{0}$ is the permittivity of free space $\left(8.85 \times 10^{-12} \mathrm{coul}^{2} / \mathrm{Nt}-\mathrm{m}^{2}\right)$, A is the area $\left(\mathrm{m}^{2}\right)$, and $\mathrm{d}$ is the charge separation distance $(m)$. Combining these expressions, rearranging, substituting $\tau$ for $d$, and defining $\sigma$ as the surface charge density $(\sigma=q / A)$ yields:

$$
\sigma \tau=\mathrm{D} \varepsilon_{0} \mathrm{Z}
$$

where $\mathrm{Z}$ has been introduced in place of $\mathrm{V}$ and is known as the zeta potential. Equation (1) defines the zeta potential $\mathrm{Z}$ (volts or $\mathrm{Nt}-\mathrm{m} / \mathrm{coul}$ ) which is an electrical potential between a fluid-wall system arising from the electrical 
double layer. The zeta potential is a property of the fluid-wall system and has typical values from 10's to 100's of millivolts. Measurements of $\mathrm{Z}$ reported in the literature are usually made with glass walls ${ }^{4}$.

The length scale $\tau$ is sufficiently large so that external pressure gradients force the fluid with its diffuse outer layer of charge to flow along the fixed wall, thus, a current is generated. This is a fluid dynamic analog to the current in a Van De Graff generator where charge mechanically moves on a non-conducting belt. The current (coul/s) due to flow induced charge assuming axial flow in a cylindrical tube of radius $R$ is:

$$
\mathrm{i}_{\mathrm{z}}=\sigma \mathrm{U}_{\mathrm{w}}(\tau) 2 \pi(\mathrm{R}-\tau)
$$

where $U_{w}(\tau)$ is the fluid velocity $(\mathrm{m} / \mathrm{s})$ at distance $\tau$, and $2 \pi(R-\tau)$ is the circumference of the flowing "shell" of charge. Substituting for $\sigma$ from Equation (1) and the expression for $U_{w}(\tau)$ for a Newtonian fluid in laminar flow gives:

$$
\mathrm{i}_{\mathrm{Z}}=\pi \mathrm{R}^{2} \mathrm{D} \varepsilon_{0} \mathrm{Z} \Delta \mathrm{P} / \mu \mathrm{l}
$$

where $\Delta \mathrm{P}$ is the pressure differential over the tube ( $\mathrm{Pa}$ or $\left.\mathrm{Nt} / \mathrm{m}^{2}\right), \mu$ is the fluid viscosity (Pa-s or $\mathrm{Nt}-\mathrm{s} / \mathrm{m}^{2}$ ), and 1 is the tube length $(\mathrm{m})$. For a fluid with electrical conductivity given by a resistance $\Re=\rho 1 / \pi R^{2}$ where $\rho$ is the fluid resistivity (ohms-m or coul $2 / \mathrm{Nt}^{2} \mathrm{~m}^{2}-\mathrm{s}$ ), a drain current forms that is equal to the generated current, thus a steady state voltage is achieved. Combining Equation (3) with Ohms Law yields:

$$
\mathrm{V}=\mathrm{D} \varepsilon_{0} \mathrm{Z} \rho \Delta \mathrm{P} / \mu
$$

where $\mathrm{V}$ is the voltage from flow induced charging. This voltage is called the streaming potential and was first described by Smoluchowski in $1903^{5}$. Equation (4) is Smoluchowski's Equation (28) caste in SI units. For fluids with low resistivity $\left(10^{2}\right.$ to $\left.10^{5} \mathrm{ohm}-\mathrm{m}\right)$ at modest pressures $\left(10^{3}\right.$ to $\left.10^{6} \mathrm{~Pa}\right)$, the streaming potential is of the order of a volt 6 . Only for fluids of very high resistivity do appreciable voltages develop.

For applications in reduced gravity where a small volume of fluid (usually silicone oil) is injected into an apparatus in the course of an experiment, it is most useful to consider the case for a non-conducting fluid where no significant drain currents exist, thus allowing the possibility of large charges. For this case the steady state voltage given by the streaming potential of Equation (4) no longer applies. Reforming the equations for this case, it is convenient to replace the pressure differential term (which is seldom known) with the volumetric flow rate $\left(\mathrm{m}^{3} / \mathrm{s}\right), \mathrm{Q}=V / \mathrm{t}=\pi \mathrm{R}^{4} \Delta \mathrm{P} / 8 \mu \mathrm{l}$, where $V$ is the volume $\left(\mathrm{m}^{3}\right)$ of fluid injected into 
the experiment over time $t(s)$. Defining the flow induced charge as $q=i_{2} t$ and by using Equation (3) gives:

$$
\mathrm{q}=8 \mathrm{D} \varepsilon_{0} Z V / \mathrm{R}^{2}
$$

where $q$ is the accumulated charge on the volume of fluid $v$. The voltage associated with this charge is estimated by assuming the fluid volume has a spherical shape and the charge is on the free surface ${ }^{7}$. The relation $V=q / 4 \pi D^{\prime} \varepsilon_{0} a$ then gives:

$$
\mathrm{V}=8 \mathrm{ZDa}^{2} / 3 \mathrm{D}^{\prime} \mathrm{R}^{2}
$$

where $\mathrm{D}$ ' is the dielectric constant of the surrounding medium and "a" is the sphere radius. The charge force exerted upon this fluid body is determined from $F=q^{2} / 4 \pi D^{\prime} \varepsilon_{0} d$ where $q$ is defined by Equation (5) and is operating on an equal magnitude image charge at a separation of $d$. For this equation to give an accurate result, the charge must be uniformly distributed over the sphere's surface and the separation distance $d$ must be greater than that of the sphere radius "a" which is not necessarily true under experimental conditions. However, this approach is adequate for determining whether flow induced charging will affect a particular experiment using the scaling arguments developed in the next section.

\section{Results Applied to Reduced Gravity}

To determine whether the force resulting from flow induced charging is significant compared to other system forces, we make dimensionless ratios or $\pi$ groups using the method of Buckingham $\pi^{8}$ and inspect fluid behavior under experimental conditions while the magnitudes of these groups change. Several forces significant in reduced gravity fluid dynamic experiments are: surface tension, viscous, inertial, and gravitational (residual). For the scope of this paper, only surface tension force is considered. For more applications, see Ref. 9.

Applying the method of Buckingham $\pi$ to surface tension force, $F=\gamma 1$, where $\gamma$ is the surface tension $(\mathrm{Nt} / \mathrm{m})$ and 1 is a characteristic length $(\mathrm{m})$, with charge force gives the dimensionless $\pi$ group:

$$
\pi=q^{2} / \gamma \operatorname{ad}^{2} D^{\prime} \varepsilon_{0}
$$

for a free volume of fluid of characteristic length "a" surrounded by a medium with dielectric constant $\mathrm{D}^{\prime}$ with the charge determined by Equation (5) at a 
separation of $d$. This $\pi$ group is the dimensionless ratio of charge force to surface tension force without the geometric constants.

To evaluate this $\pi$ group under microgravity conditions, we rely on an experiment performed by Joe Allen 10 on STS-4. A sphere of silicone oil was injected from a syringe and subsequently experienced large charge forces as it was brought in the proximity of a flat plastic plate. The sphere was about 20 $\mathrm{mm}$ in diameter and the distance at which charge effects were observed was about $10 \mathrm{~mm}$. From Equation (5) the fluid charge is calculatedto be $6 \times 10^{-10}$ coul at 520 volts $^{11}$. At this distance, the $\pi$ group from Equation (7) is 0.68 using a surface tension of $6 \times 10^{2} \mathrm{Nt} / \mathrm{m}$. At values less than 0.68 (charge separation greater than $10 \mathrm{~mm}$ ), no significant charge forces are observed. At values greater than 0.68 (charge separation less than $10 \mathrm{~mm}$ ), charge forces are significant compared to surface tension. Observations described the oil sphere as "hula dancing". We have been unable to find a suitable name for this $\pi$ group, so we propose to call it the Hula Number, $H=q^{2} / \gamma a^{2} D^{\prime} \varepsilon_{0}$. We recommend that Hula Numbers be less than 0.5 to avoid charge force artifacts.

\section{Conclusions}

Microgravity experiments on free fluid surfaces of large length scale could be subject to experimental artifact from flow induced charging. Under conditions favorable for flow induced charging, flowing liquids develop static electrical charge which manifests itself as a force whose magnitude approaches that of surface tension force. Conditions favorable for charge forces are: a nonconducting liquid, a small diameter non-conducting flow passage, a large flow volume, and a small separation distance between the fluid and another object. The static charge is calculated using Equation (5) and the dimensionless group we call the Hula Number, defined by Equation (7), compares the magnitude of charge force to surface tension force. From experimental observations, the Hula Number should be less than 0.5 to prevent artifact or unwanted fluid motion.

\section{Acknowledgments}

It is a pleasure to thank Joseph P. Allen, former NASA astronaut, for his detailed observations on microgravity fluid behavior. This work was performed under the auspices of the United States Department of Energy.

\section{References}

1. Flow induced charging has inspired the following: A liquid in motion, a flowing dielectric, forms a static charge, an electron isometric. Electrons are freed from the wall-shear tug, not unlike those developed from feet scuffing rug. This minute charge we are forced to endure, from a third order effect that is rather obscure. 
2. This has become known as "Bad Dog" behavior.

3. Adamson, A. W., Physical Chemistry of Surfaces, Wiley, 1976, pp. 196-214.

4. Kruyt, H.R., Colloid Science, Elsevier, 1952.

5. Smoluchowski, M. Marie, "Przyczynek do teoryi endosmozy elektrycznej i niektorych zjawisk pokrewnych", Bull. Int. DE L'ACADEMIE DES SCIENCES, No 3, Mar. 1903, pp. 182-199.

6 Although for this example, sparks will not fly, this low voltage over long periods of time can contribute to electrochemical corrosion.

7. Equation (5) assumes no final geometry for the fluid but to determine the voltage as in Equation (6), a geometry much be chosen. For a non-conducting fluid such as silicone oil, the charge is not necessarily on the free surface like we are use to thinking with a conductor. Pockets of charge have been observed which cause small blobs to be taken from within the volume, torn through the interface, and splashed against some unsuspecting surface in classical "Bag Dog" behavior.

8. Buckingham, E., Phys. Rev., 2, (1914) p. 345 ; Welty, J.R., Wicks, C.E., and Wilson, R.E., Fundamentals of Momentum. Heat, and Mass Transfer, Wiley, 1984, pp. 154-156.

9. Pettit, D.R., "Flow Induced Charging in Liquids: Applications to Fluid Dynamic Experiments in Microgravity", to be submitted to Phys. of Fluids 1996.

10. Allen, J.P., personal communications.

11. For these calculations, $D=5$ (silicone oil), $D^{\prime}=1$ (air), $R=0.5 \mathrm{~mm}$ (syringe tip), $a=10 \mathrm{~mm}$ (radius oil sphere), and $\mathrm{Z}=100 \mathrm{mV}$. A measured value for the zeta potential of silicone oil on polyethylene was not found. This value is an estimate based on values for organic based oil on glass. 